\section{Digital dental photography. Part 10: printing, publishing and presentations}

\author{
I. Ahmad'
}

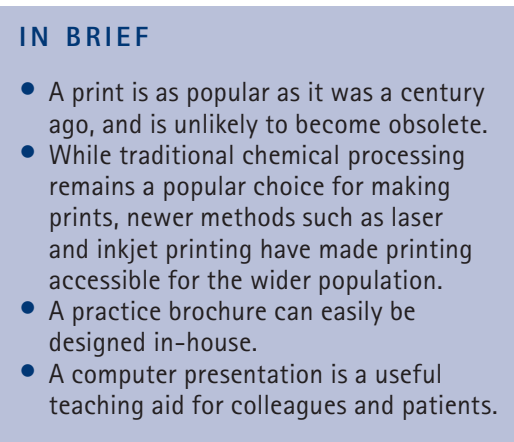

- A print is as popular as it was a century ago, and is unlikely to become obsolete. While traditional chemical processing remains a popular choice for making prints, newer methods such as laser and inkjet printing have made printing accessible for the wider population. designed in-house.

teaching aid for colleagues and patients.

The final part of this series on digital dental photography details how to use images to their maximum potential. The purpose and uses of dental photography have previously been covered in Part $2{ }_{1}^{1}$ and the ensuing discussion concentrates on the technical aspects of printing, publishing and audio-visual presentations.

\section{PRINTING}

Before the advent of digital photography, the only method of viewing a picture was after it was developed and printed. This is no longer the case with digital images, which can be instantly viewed on builtin LCD camera displays or by transferring them to a computer. However, for many applications, printing on photographic paper is still preferred and indeed, is very popular. A print can be easily viewed by many people, posted and transported with ease. In addition, printed images are essential for memorable events such as

\section{FUNDAMENTALS OF DIGITAL DENTAL PHOTOGRAPHY \\ 1. Digital dental photography: an overview \\ 2. Purposes and uses \\ 3. Principles of digital photography \\ 4. Choosing a camera and accessories \\ 5. Lighting \\ 6. Camera settings \\ 7. Extra-oral set-ups \\ 8. Intra-oral set-ups \\ 9. Post-image capture processing \\ 10. Printing, publishing and presentations}

${ }^{1}$ General Dental Practitioner, The Ridgeway Dental Surgery, 173 The Ridgeway, North Harrow, Middlesex, HA2 7DF

Correspondence to: Irfan Ahmad

Email: iahmadbds@aol.com

www.IrfanAhmadTRDS.co.uk

\section{Refereed Paper}

Accepted 15 November 2008

DOI: $10.1038 /$ sj.bdj.2009.814

${ }^{\circ}$ British Dental Journal 2009; 207: 261-265 birthdays, weddings or ceremonial functions. Therefore, the printed image, unlike film, is not and probably will never become obsolete. Today, there are many methods available for printing a photograph including traditional chemical processing, laser, inkjet and thermal sublimation printing.

\section{Chemical processing}

Chemical processing still remains a popular option for making photographic prints. Although chemicals and techniques have been refined, the basic principles are identical to those used more than a century ago. The procedure is as follows. A paper coated with light-sensitive dyes is exposed by either light or lasers to leave an 'impression' of the image. The sensitised paper is then developed by a series of chemicals to 'reveal' the image. The process is identical to developing radiographs using developer and fixer solutions. The ubiquitous photo-minilab outlets automate this process, delivering prints in less than an hour. This is a very cost effective and economical method for making prints of an acceptable quality of 300 to 400 dpi (dots per inch). However, if an entire camera memory card is sent for printing without prior vetting on a computer screen, substantial wastage is highly probable since some prints may be unacceptable and subsequently discarded. In order to avoid this wastage, a prudent approach is to view the images on a computer monitor and compile a selection before forwarding the memory card, or other storage media, to a minilab for printing.

\section{Laser printing}

This process involves a laser beam creating an electrostatic charge on a drum for the exposed parts of an image. The charged drum then attracts the powered toner and transfers it onto the paper. Pressure and heat is applied to ensure that the toner powder tenaciously adheres to the paper. Although the price of both black-and-white and colour printers are rapidly decreasing, the price of replacement toner cartridges is relatively high. The quality of the prints depends on the equipment specifications, but the major drawback is that only plain paper can be used due to the inherent process of applying heat and pressure to prevent the toner lifting off the paper. For this reason, laser printing has not gained popularity for photographic applications since true photographic or textured papers cannot be used.

The main advantage of laser printers is speed, making them ideal for letter writing and incorporating clinical images for specialist advice or referral. Both digital images and radiographs are easily cut and pasted into word processing software, which is invaluable for depicting pathology or other prevailing clinical situations.

\section{Inkjet printing}

If the quality of a laser print is unacceptable, the next option for in-house printing is using an inkjet printer. Inkjet printers use single or multiple cartridges that deposit droplets of ink onto the receiving paper. The droplets are extruded from the cartridge either by heat or piezoelectric 


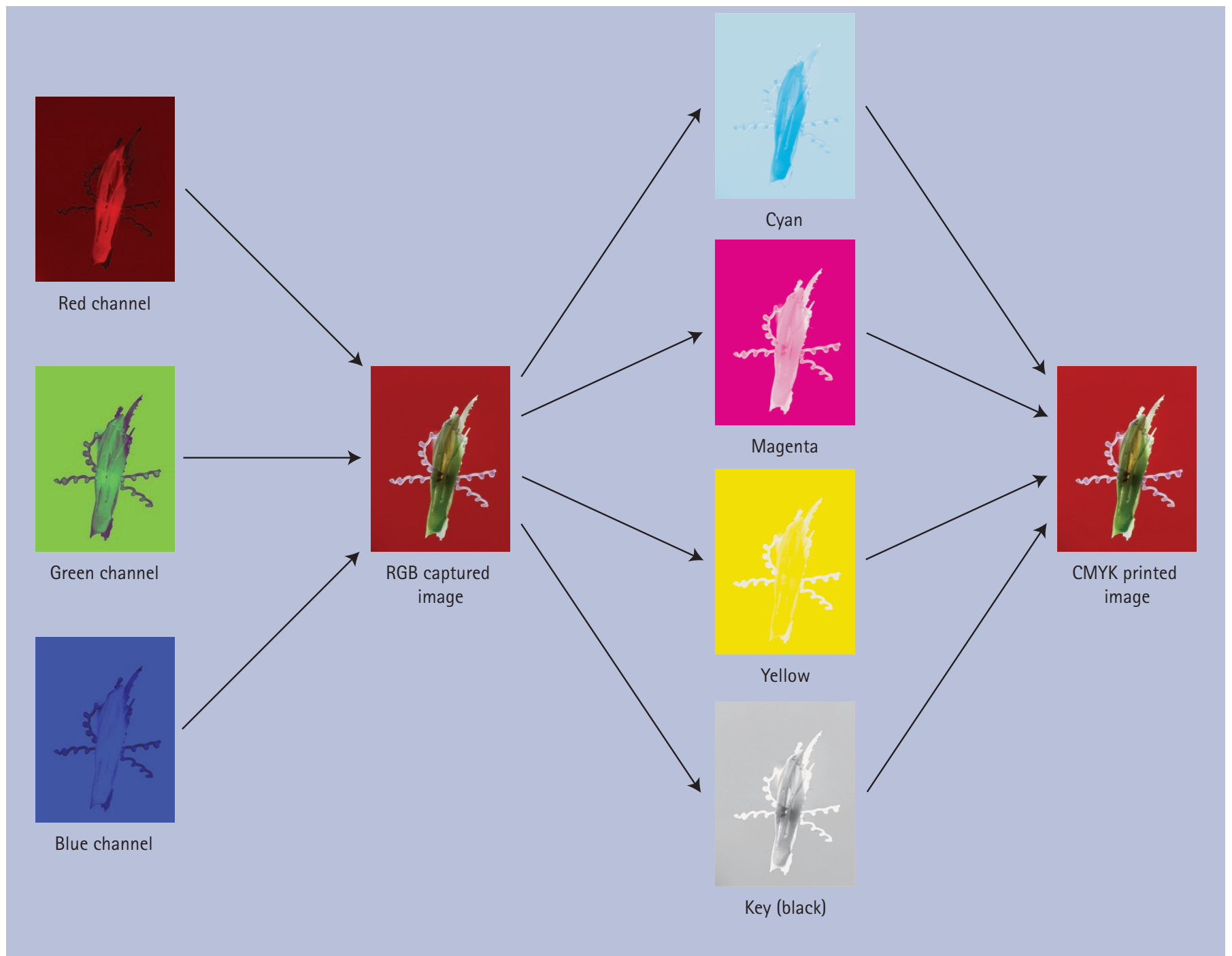

Fig. 1 Schematic representation of RGB image capture and CMYK printing

elements. Newer printer models offer ports for inserting camera memory cards as well as wireless connection from cameras or computers. Although plain paper can be used, the endearing feature is that photographic as well as textile media can be used. This offers vast latitude and the results are identical or even superior to conventional chemical processing. The image resolution often exceeds $4,800 \times 1,200$ dpi using either RGB or CMYK colour printing modes. The selection of photographic paper is enormous, with a range of different textures and surface finishes, for example canvas, matte, gloss, etc. Also, the sizes of the prints can range from the familiar $5 \times 4$ print to $\mathrm{A} 1$ posters. Inkjet printers are extensively used for printing pictures at home, as well as by professional photographers working in studios. The cost of an inkjet printer is insignificant compared to the ink cartridges and quality photographic paper.
Therefore, the expensive refill cartridges and paper usually offset any savings on purchasing the printer. There are numerous retail outlets offering to refill cartridges at nominal prices, but this is a false economy and usually counterproductive. Firstly, if the cartridge leaks during use, the picture and printer are irreparably damaged. Secondly, proprietary inks contain a lacquer that protects the print from mechanical damage and prevents colour fading due to exposure to light, which may be absent from cheaper third party refilling inks.

Inkjet printers in a dental surgery offer quality prints in minutes, which can be used for communication between dental colleagues, patients and dental technicians or included in a referral letter to a specialist. Furthermore, larger prints can be used to build a portfolio of different treatment modalities for patient and staff education, or even posters for in-house marketing.

\section{Thermal sublimation and thermal transfer printers}

The technology employed by thermal printers consists of a donor band housing the dyes, either RGB or CMYK, which is released onto special receiving paper by the application of heat. The resolution is acceptable at $300 \mathrm{dpi}$, and some equipment also seals the print. The advantage of these printers is their compactness, portability and direct connection to digital cameras or ports for accepting memory cards, thereby by-passing a computer. The selling point of thermal printers is expedience at a modest price. The Kodak P712 dental camera uses thermal printing with its EasyShare docking port printer for instant prints.

Thermal printers have similar uses in dentistry as inkjet printers but without the flexibility of using different types of paper.

\section{PUBLISHING}

There are many occasions when in-house 
desktop publishing is sufficient for printing circulars or memos, but when large volumes of practice stationery, brochures or newsletters are required, it is cost effective to use a printing house.

There are four main types of printing processes, relief, intaglio, offset lithography and screen. Before an image can be printed using a printing press, the additive RGB colour mode that captured the picture must be converted into CMYK subtractive colour mode (Fig. 1). This conversion process is termed colour separation. The subtractive colours are cyan, magenta and yellow, with black representing the key colour, forming the acronym CMYK. Two printing process are available, threecolour printing, which requires colour conversion only into the three subtractive colours, CMY, or four-colour printing that requires the addition of black, CMYK. The conversion process is crucial for ensuring that colours are reproduced as accurately as possible to correspond to the original in the RGB mode. It is also worth remembering that conversion from RGB to CMYK for printing will diminish the dynamic range and alter the colour space. The RGB mode enjoys both larger dynamic ranges and colour spaces that the printing process lacks, and if separation is inadequately performed the printing image will lose the vitality and quality of the original. The process of colour separation is complex, and unless one has the experience or training, is best assigned to a lithographer.

\section{Relief printing}

Relief printing was the first printing process developed, using woodcuts or metal plates that were raised and coated with ink to be deposited onto paper, hence the name relief printing. Nowadays, wood and metal are replaced with plastic materials, the so-called flexography.

\section{Intaglio or gravure printing}

Instead of having raised areas, as in relief printing, in intaglio printing the printable parts are recessed and soak ink from a well and then deposit it onto the printing paper.

\section{Offset lithography printing}

Offset lithography or 'litho' is the most popular and widely used method for printing today. Similar to earlier printing methods, the portions to be printed are treated to accept oil-based inks, while the remainder of the plate accepts water-based dyes. The reason for the term 'offset' is because the paper does not directly make contact with the plates but instead contacts a rubber blanket, which collects ink that is subsequently transferred to the paper.

\section{Screen printing}

This process uses fine meshes such as silk cloths that are blocked out for the nonprintable areas, while the naked parts allow ink to squeeze through the mesh and onto the paper. This form of printing is creative, allowing many artistic effects, and was used with tremendous vigour by the 1960s artist Andy Warhol to create his iconic works of art.

The choice of printing method depends on the printing house and the need of the client. As mentioned above, litho printing is the most prevalent with many standardised protocols. For example, for business cards of a small printing run, professional digital laser printing is cheaper than litho. On the other hand, for large runs of brochures with text, drawings and images, litho is more economical.

\section{Publishing a practice brochure}

Publishing a practice brochure is a good example to illustrate the steps involved in the printing process. The first stage is designing. This can be assigned to a graphic house or easily carried out in-house using a variety of inexpensive layout software, which allows greater control and can be an exciting task. Most drawing and word processing software are shipped with numerous templates for popular stationery items such as letterheads, businesses cards, brochures and newsletters (Fig. 2). The templates have placeholders for images and text, and the desired images are simply cut and pasted and the software crops or scales them to fit the allotted placeholder size. Next, text is typed into the assigned boxes, for example, headings, sub-heading and body text. Once the design is finalised, the file is saved in the propriety software file format (Fig. 3). Because of the abundance of graphics applications on the market, the software used to design the brochure may not be the same as the one used by the

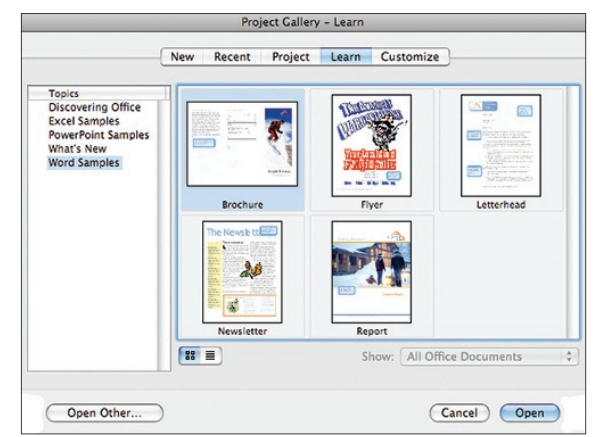

Fig. 2 Templates for popular stationery items in a word processing software package

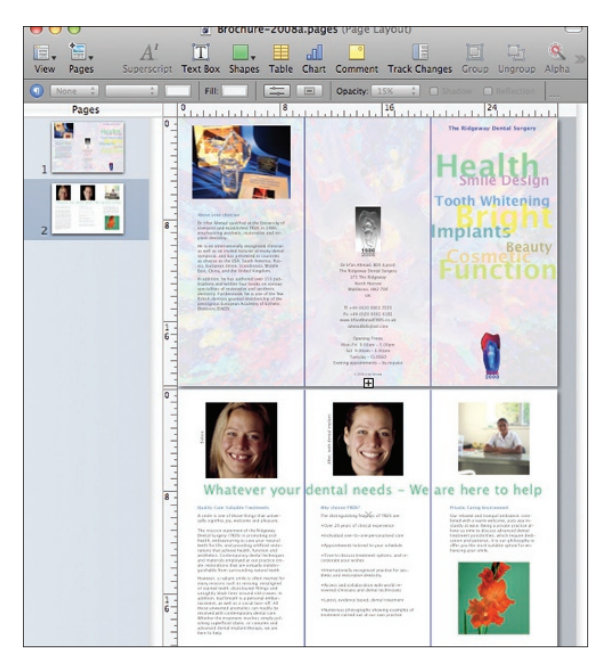

Fig. 3 The completed brochure design and layout is first saved in the propriety software file format

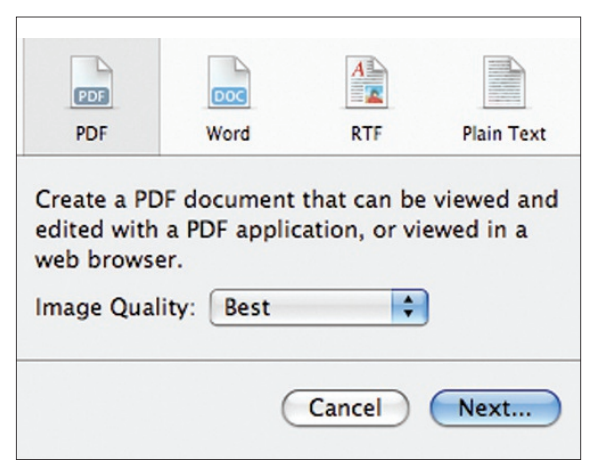

Fig. 4 Exporting a file to a 'best quality' PDF format

printing house. Also, if the design software does not allow colour separation into CMYK for four-colour printing, the file will need to be exported into a type that allows this facility. One option is to export the file in a PDF file format using a high quality mode, and then forward it to the printing house (Figs 4-6). The lithographer can open and edit the PDF file before proceeding to colour separation and preparing a digital proof.

The ultimate goal is trying to approximate as closely as possible the colours in 


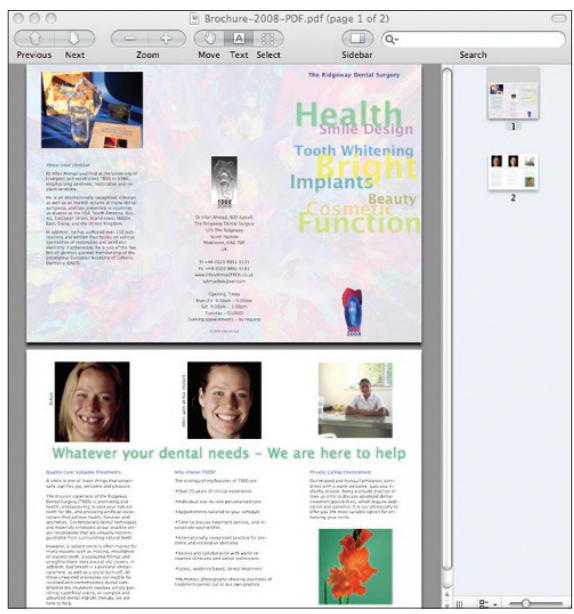

Fig. 5 PDF conversion of original file

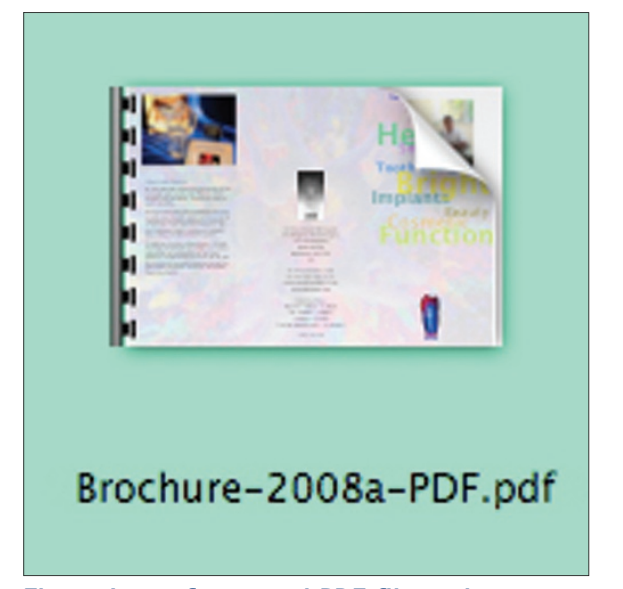

Fig. 6 Icon of exported PDF file, to be forwarded to the printing house

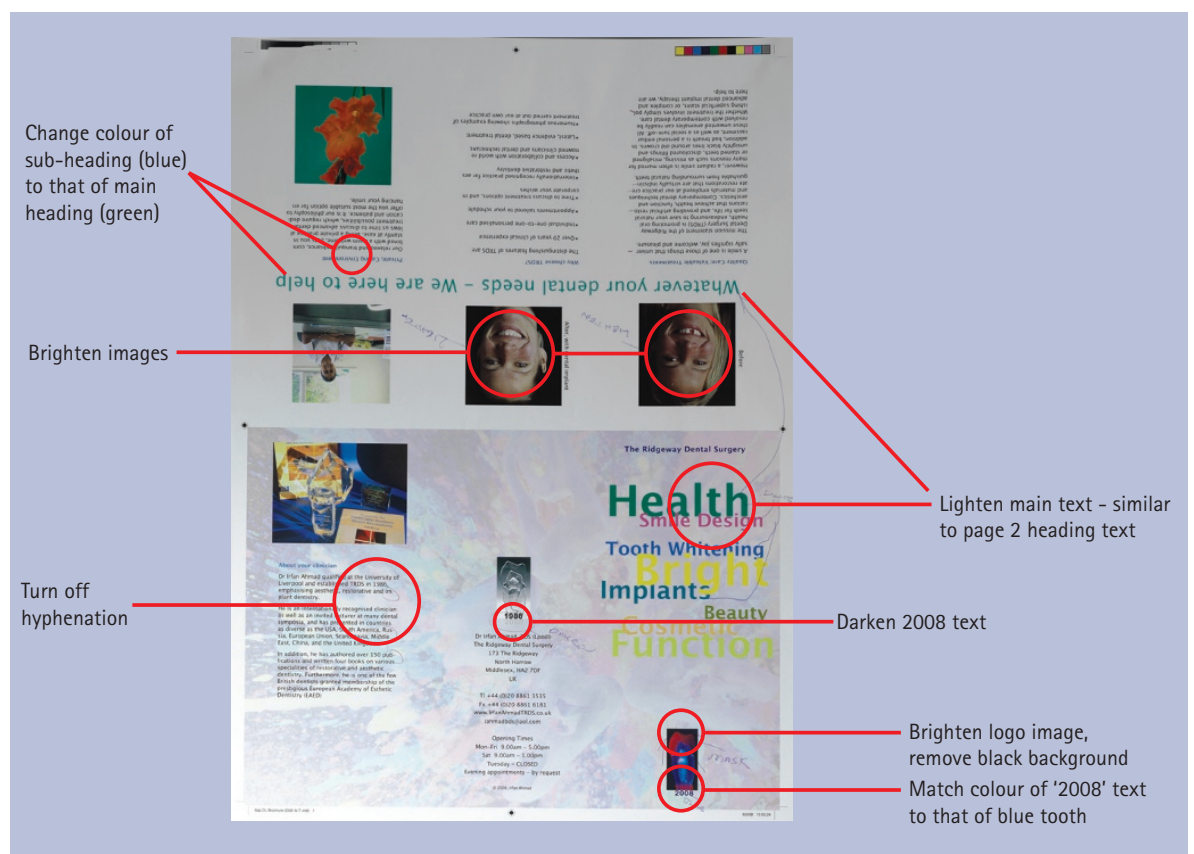

Fig. 7 First digital proof of a practice brochure showing errors that require amending

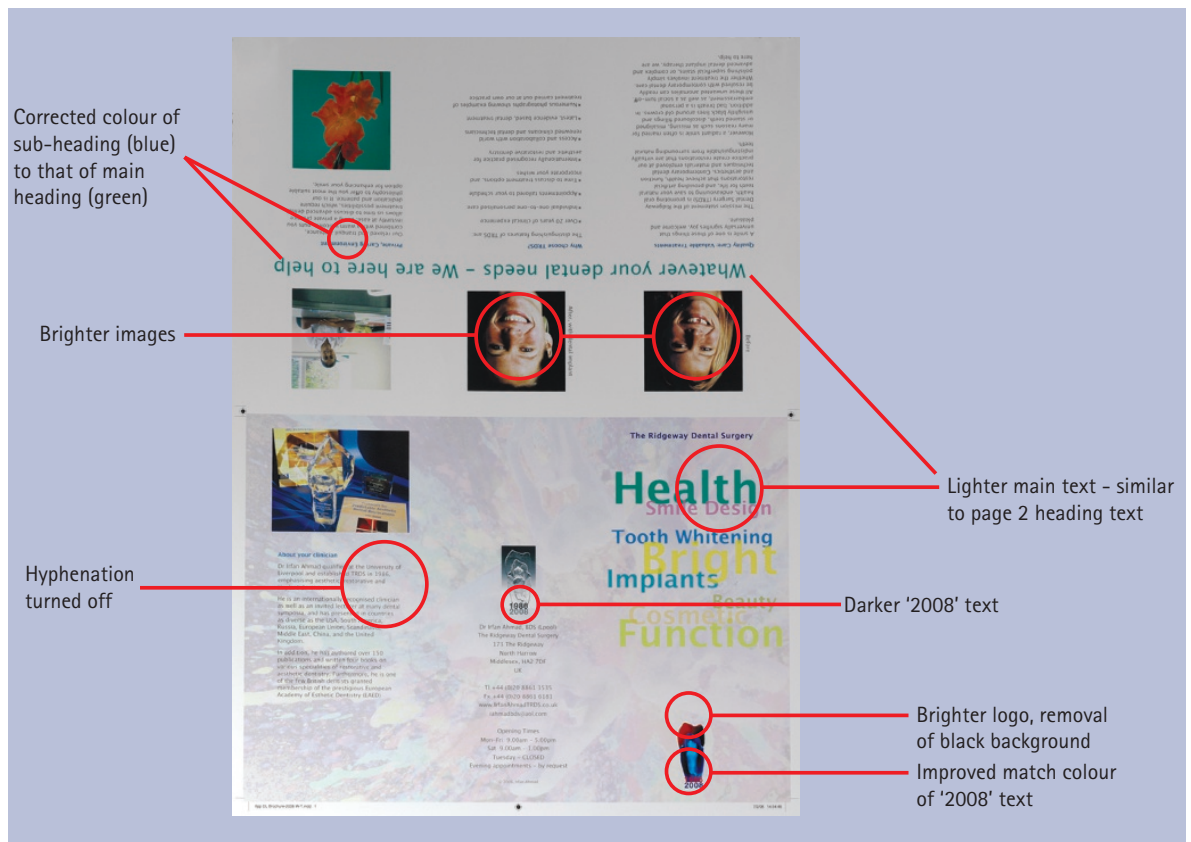

Fig. 8 Second digital proof of practice brochure incorporating the necessary changes

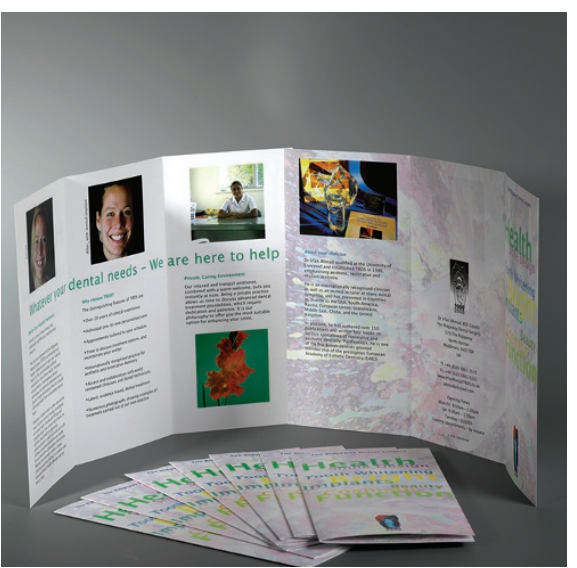

Fig. 9 The printed practice brochure

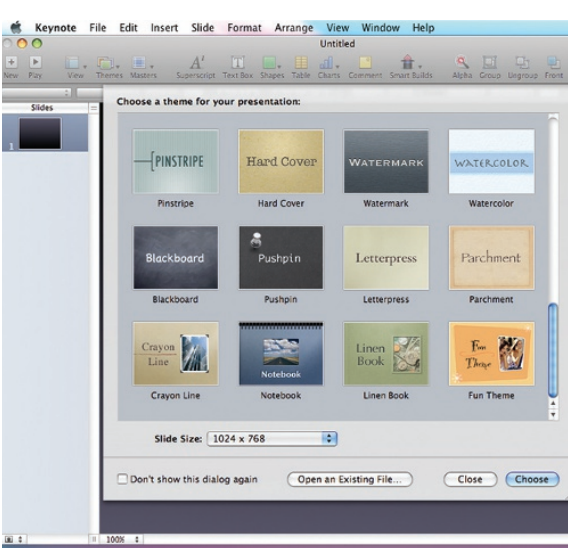

Fig. 10 Keynote $^{\mathrm{TM}}$ is a presentation software package exclusive to Apple Mac computers

the CMYK printed images to that of the original RGB capture. In reality, this task is rarely achieved unless one spends vast amounts of money for colour corrections by employing a colourist to match CMYK to RGB both subjectively and objectively by using colour-measuring devices such as colorimeters. Furthermore, since potential readers of the brochure will rarely see the original RGB image for comparison, a CMKY approximation suffices. There are instances where practice logos, drawings or parts of images require precise colour reproduction. If this is the case, a spot colour can be added in addition to the CMYK processed colours. There are many colour selection charts and wheels available from printing houses, for example Pantone ${ }^{\varpi}$, for choosing specific spot colours. In theory, the number of additional spot colours is limitless so long as the budget allows for these extravagances.

It is always advisable to order a digital proof for visualising the layout, reviewing text, checking spelling, and gauging the approximate colour rendition of the images and any coloured text and 


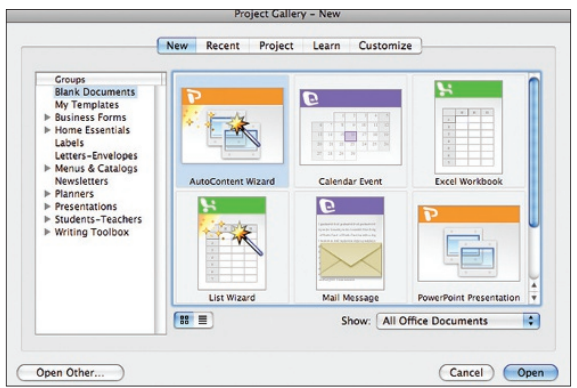

Fig. 11 A good starting point for creating a presentation is choosing the PowerPoint Wizard for a step-by-step guide through various stages

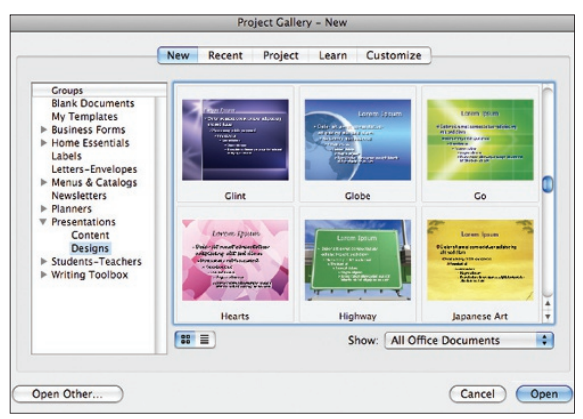

Fig. 12 PowerPoint offers an innumerable choice of templates

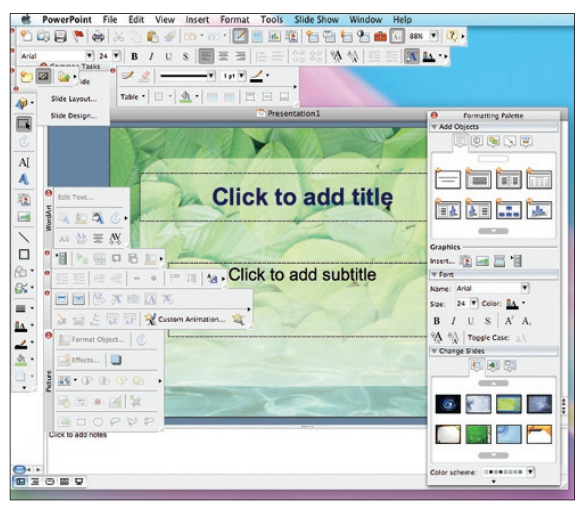

Fig. 13 PowerPoint is a powerful application, capable of sophisticated presentations drawings. As well as revealing overlooked mistakes at the designing stage, a proof also allows scrutiny of the anticipated resolution of the images, drawings and text (Fig. 7). If changes are necessary, these are amended in the software and a second proof requested (Fig. 8). It is also advisable to ask other members of the dental team to view and proof-read the document with a 'fresh pair of eyes' for errors and omissions. Alternately, one can employ the services of a professional proof-reader. Once approval is forthcoming, the type of paper for the stationery is chosen and the print run is initiated (Fig. 9).

\section{PRESENTATIONS}

In addition to lecturing to fellow colleagues, building an audio-visual presentation is an ideal tool for patient and staff education, as well as promotional purposes. There are many types of software for making slick and stylish presentations which can incorporate video footage, music and narration. If music is added, it is essential to obtain the permission of the artist or the record company. Illicit use of music or images is an infringement of copyright or intellectual property and could result in litigation.

Over the last decade, Microsoft ${ }^{\circledast}$ PowerPoint $^{\mathrm{TM}}$ has become the industry standard as the presentation software of choice. Newer versions of this software incorporate an ever-increasing number of audio-visual effects, and their use is limited only by the imagination. Another software package exclusive to Apple $\mathrm{Mac}^{\circledast}$ computers is $\mathrm{Keynote}^{\mathrm{TM}}$, which is similar to PowerPoint, offering powerful tools for building creative audio-visual presentations (Fig. 10). A pitfall to avoid is trying to incorporate too many animations within a single presentation. While animated effects are dramatic, it is advisable to use them sparingly. Having a disproportionate number of acrobatic animations is visually annoying and distracting, often to a point that the message being conveyed is rejected.

The best starting point when creating a presentation is either to choose a predefined template or to use a PowerPoint Wizard that guides the operator through the stages for making a presentation (Figs 11-12). All the included templates are designed by professional graphic artists, with pleasing colour combinations rather than haphazard lurid chromatic orgies! Also, similarly to templates for stationery, PowerPoint templates have placeholders for text and images. The images are cut and pasted and text typed into the assigned boxes. The font sizes of the headings and sub-headings are predefined and are proportionate and helpful for a visually pleasing layout. In a few hours, a simple presentation can be created, for example showing the clinical stages for a particular treatment modality such as fillings, crowns, implants, etc. If one is adventurous and patient, PowerPoint is a powerful application capable of very sophisticated presentations and a little training with an expert is invaluable for creating stunning and enticing graphics (Fig. 13).

1. Ahmad I. Digital dental photography. Part 2: purposes and uses. Br Dent J 2009; 206: 459-464. 